\title{
Efectividad a largo plazo de los tratamientos de mantenimiento con metadona en personas con adicción a derivados opiáceos
}

\section{Long term effectiveness of methadone maintenance treatments in persons with addiction to opiates}

\section{Julio Bobes Garcia*; María Teresa Bobes Bascarán ${ }^{* *}$}

*Área de Psiquiatría, Departamento de Medicina. Universidad de OviedoCIBERSAM

**Departamento de Personalidad, Evaluación y Tratamientos Psicológicos. Universidad de Valencia-CIBERSAM

Enviar correspondencia a:

Julio Bobes García

Área de Psiquiatría. Facultad de Medicina.

c/ Julián Clavería, s/n, 33006

Oviedo (España)

bobes@uniovi.es

\section{Resumen}

Los estudios prospectivos de grandes series con distintas dosis de mantenimiento y marcadores distales estandarizados no existen, sin embargo actualmente existe evidencia acerca de la efectividad de la metadona como tratamiento a largo plazo de la adicción a opiáceos. Entre sus resultados se puede destacar un menor consumo de sustancias ilegales, menor implicación en actividades criminales y mejor funcionamiento social general. Por otra parte, la salud física y mental permanece más conservada y recuperada. Puesto que los pacientes en tratamiento con metadona presentan menos probabilidad de padecer problemas cardiovasculares, $\mathrm{VIH}$, hepatitis $\mathrm{C}$, hepatitis $\mathrm{B}$ y otros procesos infecciosos, así como una menor comorbilidad psiquiátrica. Este editorial pretende definir y guiar el posicionamiento terapéutico a la hora de abordar el manejo de personas con abuso o dependencia a opiáceos. Para fundamentar las pautas guía se han tenido en cuenta y revisado los principales estudios a largo plazo, análisis sistemáticos y aproximaciones meta-analíticas de la efectividad de la metadona. Por todo ello se recomiendan, con el máximo nivel de evidencia, los tratamientos de mantenimiento con metadona a pesar de las debilidades en cuanto a toxicidad y riesgo cardiovascular, y las dificultades de sedación y disminución del funcionamiento cognoscitivo, siempre teniendo en cuenta la historia clínica, el estado de salud general, y los deseos y preferencias del paciente.

Palabras clave: tratamiento a largo plazo con metadona, tratamiento de mantenimiento con metadona, adicción a opiáceos, efectividad de la metadona, dosis y toxicidad de la metadona.

\section{Abstract}

There is no large prospective cohort studies using different therapeutic doses and standardized distal outcomes, however there is a fair amount of evidence on the effectiveness of methadone as a long-term treatment for the addiction to opiates. Strengths include less illegal drug use, decreased criminal activity and better general functioning. Also physical and mental health seems to be more preserved and recovered. Since patients undergoing methadone treatment exhibit diminished likelihood of suffering from cardiovascular conditions, HIV, HCV, HBV and other infections, and lesser psychiatric comorbidity. This Editorial intends to define and guide the therapeutic statement to address the management of persons with opiate abuse or dependence. These proposals have been grounded in the main long-term studies, systematic analysis and meta-analytic approaches on the effectiveness of methadone. Thereby it is recommended, with the maximum level of evidence, methadone maintenance treatments in spite of its weaknesses (ie. Toxicity, cardiovascular risk, sedation problems and cognitive impairment), considering the clinical history, general health status, and willingness and preferences of the patient.

Key words: methadone long-term treatment, methadone maintenance treatment, opiate addiction, effectiveness of methadone, doses and toxicity of methadone. 
L a adicción a los opiáceos se considera una enfermedad crónica recurrente, que conlleva graves consecuencias médicas y psicosociales a largo plazo. Sus efectos devastadores han sido bien descritos por la comunidad científica, encontrándose tasas de comorbilidad y muerte prematura de hasta 13 veces más que en la población general (Maremmani y Gerra, 2010). La salud física general de las personas consumidoras o exconsumidoras suele estar deteriorada por efectos directos o indirectos de las drogas. Asi se ha comprobado que las personas que se inyectan la droga tienen hasta casi 11 veces más probabilidades de contraer el VIH (Bao et al., 2012) y casi el doble de probabilidades de presentar una enfermedad oncológica (Randall et al., 2011). Un estudio longitudinal realizado en nuestro país con un seguimiento a 25 años, describe porcentajes de $47,2 \%$ de VIH y de hepatitis $B$ o $C$ en un $81,1 \%$ de la muestra (Jimenez-Trevino et al., 2011). En cuanto a la salud mental, casi un $80 \%$ de los consumidores han sido diagnosticados al menos de otro trastorno psiquiátrico, lo que les hace proclives a practicar mayores conductas de riesgo, a consumir otro tipo de drogas no opiáceas (Schafer et al., 2010), y por tanto, a complicar la posible intervención terapéutica.

El hecho de que se trate de una enfermedad crónica, acompañada por frecuentes recaídas, pone de manifiesto la importancia de la implementación de tratamientos a largo plazo. Este tipo de abordaje es especialmente relevante si se tiene en cuenta que los pacientes que abandonan el tratamiento suelen tener más recaídas, mayor tasa de mortalidad que aquellos que permanecen (Kleber, 2008). A este respecto, se debe tener presente que a pesar de que las revisiones sistemáticas acerca de la desintoxicación farmacológica muestran que la gravedad de la abstinencia se puede reducir, la mayor parte de los pacientes recaen, por lo que las mayores tasas de éxito se conseguirán si se aplica una intervención de cuidados psicosociales coadyuvantes (Nosyk et al., 2012).

El tratamiento de mantenimiento con metadona (TMM) es la opción farmacológica más utilizada para la adicción a opiáceos en todo el mundo, y se estima que al menos un millón de personas reciben actualmente dicho tratamiento. Esta expansión hace que sea el tratamiento que mayor grado de recomendación ha recibido, y por tanto, su eficacia y efectividad son bien conocidas (Bobes-Bascaran, Bascaran, Saiz, Garcia-Portilla y Bobes, 2012). A continuación se detallan algunos de los aspectos más relevantes que se han descrito acerca del TMM.

\section{Marcadores psicosociales del TMM}

Las tasas de empleo de los pacientes adictos a opiáceos mejoraron tras la instauración del TMM (Fernandez Miranda, 2005). Por otra parte, parece que el consume de sustancias ilegales tiende a disminuir (Zapata, Torres de y Montoya, 2012) y un aumento en el consumo de alcohol, tabaco y benzodiacepinas (Arroyo et al., 2000; Coviello, Zanis, Wesnoski, Lynch y Drapkin, 2011). En cuanto a la comisión de actividades ilegales, los estudios muestran que existe una gran reducción en las tasas de incriminación (Oliver et al., 2010) y de reincidencia (Dolan et al., 2005) de los pacientes en TMM. Respecto a la calidad de vida, los pacientes muestran peores indicadores comparados con controles sanos (Iraurgi Castillo, 2007) pero se ha demostrado que mayores dosis y más permanencia en tratamiento predice la mejoria de este marcador (Wang et al., 2012). Un estudio realizado en nuestro país muestra como una mayor satisfacción del usuario y mayor calidad de vida, están estrechamente relacionadas con el número de horas que el centro dispensa metadona, el número de pacientes por centro, la frecuencia percibida de la información que se recibe acerca de los cambios de dosis, la influencia percibida de tales cambios y la disfunción social (Trujols et al., 2011).

\section{Marcadores de salud física y mental en TMM}

Respecto a los indicadores de salud física y mental, un estudio longitudinal con un seguimiento de 30 años explora estos marcadores diferenciando por sexo, y concluye que los hombres que habian consumido el año previo referian peor funcionamiento físico y menor dolor corporal que los no consumidores, mientras que las mujeres con consumo durante el año anterior informaban que tenían peor salud mental que las mujeres no consumidoras (Grella y Lovinger, 2012). La prevalencia de comorbilidad psiquiátrica entre los sujetos dependientes de opiáceos que acuden a tratamiento varía entre $44 \%$ y $93 \%$, siendo los trastornos depresivos y de ansiedad y los trastornos de personalidad antisocial y límite los más frecuentes (Mateu, Astals y Torrens, 2005). Un estudio reciente, señala como aquellos pacientes con trastornos psiquiátricos más graves mejoran significativamente en las dimensiones psicológicas y psiquiátricas del SCL-90 durante el tratamiento con metadona (Pani et al., 2011).

Las tasas crudas de mortalidad de los pacientes en TMM se han estimado en torno a 1,49/100 al año, siendo la muerte súbita, la sobredosis y el cáncer las principales causas de muerte (Rosca et al., 2012). De todas maneras, varios metaanálisis muestran como los pacientes en TMM presentan una mayor disminución de las conductas de riesgo para el VIH, menor riesgo de sobredosis mortal (Torrens Melich y Fonseca Casals, 2009) y menores tasas de mortalidad que los pacientes que abandonan tratamiento (Fernandez Miranda, 2005). En conclusión, la metadona ha demostrado que produce beneficios físicos pero también se ha asociado con cardiotoxicidad y riesgo de arritmia por lo que debe considerarse la discontinuación o reducción de la dosis si en el ECG pretratamiento, el intervalo QTc excede los 500 mseg (Soyka et al., 2011).

\section{Dosis recomendadas}

Este continúa siendo uno de los temas más controvertidos en el TMM, puesto que las dosis empleadas en los ensayos clínicos difieren de aquellas utilizadas en la práctica clínica y no se han podido realizar estudios comparativos de 
largo plazo (años) con diferentes dosis dirigidos a recomendar una dosis a muy largo plazo (más de 2 años). Parece que dosis más bajas de metadona conllevan una peor efectividad, y la dosis media óptima en la que los efectos clínicos se pueden percibir ronda los $60 \mathrm{mg}$ (Amato et al., 2005). La Federación Mundial de Sociedades de Psiquiatría Biológica (WFSBP) ha emitido un documento reciente en el que se concluye que dosis más elevadas se asocian con mejores tasas de retención e indicadores de tratamiento, y que los pacientes con trastornos psiquiátricos comórbidos son candidatos a beneficiarse de dosis mayores a $100 \mathrm{mg} / \mathrm{dia}$ (Soyka et al., 2011). Por tanto, la dosis media estimada de metadona deberá comprender un rango de entre 60-100 mg diarios, recomendando un inicio gradual con dosis menores para evitar los riesgos asociados a la introducción de metadona (Lingford-Hughes, Welch, Peters y Nutt, 2012). En cuanto a la reducción del TMM, se ha comprobado que aquellos pacientes con reducciones de dosis más graduales, mostraban mayor porcentaje de éxito a largo plazo (Nosyk et al., 2012).

\section{Seguridad de los TMM}

Los aspectos de seguridad deben considerarse y sopesarse, teniendo en cuenta los beneficios con respecto al consumo de droga ilegal continuado, con mayor mortalidad asociada. A este respecto, se ha descrito que los tratamientos con metadona salvan dos vidas por cada fallecimiento anual (Whelan y Remski, 2012). La fase de instauración inicial es especialmente peligrosa, puesto que el riesgo de sobredosis mortal durante las primeras dos semanas de tratamiento es casi 7 veces mayor que aquella estimada para pacientes dependientes a la heroína que no reciben tratamiento y hasta 98 veces mayor que los pacientes con dosis de mantenimiento de metadona (Nosyk, Marsh, Sun, Schechter y Anis, 2010).

Siguiendo unas recomendaciones elaboradas en nuestro país respecto a los PMM (Martinez Delgado, Fernández-Repeto Guilloto y Ramírez López, 2005) hay que tener en cuenta que la dosis óptima de metadona en la fase de inducción o estabilización es aquella que previene la aparición del sindrome de abstinencia durante al menos 24 horas, reduce o elimina la apetencia el craving y bloquea los efectos euforizantes de cualquier opiáceo autoadministrado de manera ilegal. Por tanto, dadas las diferencias individuales interpersonales, la farmacoterapéutica deberá ser inducida para cada caso particular, con dosis iniciales de entre 20-50 mg. metadona/dia, salvo excepciones, para evitar el riesgo de sobredosis. Posteriormente la fase de mantenimiento y/o deshabituación, que comenzará a partir del final del primer mes, se prolongará el tiempo necesario que permita el funcionamiento óptimo biopsicosocial de cada persona, especialmente se tendrá en cuenta, el impacto sobre el ritmo cardíaco y el estado de salud general del paciente. El riesgo de efectos cardíacos (prolongación del intervalo QTc) resulta mayor en los mantenimientos con metadona, y se asocia generalmente, a las dosis altas (> $100 \mathrm{mg} / \mathrm{dia}$ ). En un estudio de prevalencia de la prolongación del intervalo OT durante el tratamiento de mantenimiento con metadona en un $4,6 \%$ de los pacientes el OT era mayor que 500 mseg y el $28,9 \%$ presentaba prolongaciones del QT mayores que 450 mseg (Anchersen, Clausen, Gossop, Hansteen y Waal, 2009).

\section{Líneas futuras de mejora de los TMM}

Gran parte de los estudios de investigación adolecen de no tener diagnósticos bien ajustados (Arango y Bobes, 2012; Reed y Ayuso-Mateos, 2011) buenas evaluaciones basales estandarizadas, en las que se incluya la gravedad de la adicción (Casares-Lopez et al., 2011; Diaz-Mesa et al., 2010), aspectos diferenciales de género, entorno socio-cultural (ej. España, Latinoamérica), situación psicosocial y económica, la comorbilidad física y psiquiátrica, particularmente de trastornos de la personalidad. La investigación a largo plazo con la que contamos, adolece de la utilización estandarizada de indicadores o marcadores distales de resultados de tratamiento como dosis, pautas, toxicidad cardiovascular, nivel de discapacidad, calidad de vida, problemas legales, funcionamiento global, estado de salud física o nivel de integración socio-laboral. Además, dados los problemas inherentes a esta adicción, el seguimiento longitudinal de los pacientes se hace especialmente difícil, por lo que se necesitan más estudios a largo plazo, que no sólo incluyan pacientes en tratamiento sino toda la población de consumidores de opiáceos.

Por otra parte, a pesar de que la mortalidad representa unos de los efectos principales del abuso de la sustancia, esta variable no se suele especificar en los ensayos clínicos, y tampoco suele emplearse como marcador de eficacia del tratamiento, por lo que debería analizarse junto con el impacto del consumo supervisado como medida para prevenir la sobredosis ("National Institute for Health and Clinical Excellence. Methadone and buprenorphine for the management of opioid dependence," 2007).

Respecto al tratamiento psicosocial combinado con el tratamiento de mantenimiento con metadona, a pesar de que una revisión de la Cochrane indicaba sus efectos beneficiosos, parece que no existe suficiente evidencia acerca de la reducción del consumo de heroína o de pacientes abstinentes durante el seguimiento (Amato, Minozzi, Davoli y Vecchi, 2011) lo que pone de manifiesto la necesidad de diseñar programas específicos para este tipo de adicción encaminados a lograr una mayor normalización del paciente en todas las esferas de su vida.

Finalmente, respecto a los propios programas de mantenimiento con metadona, se deben superar ciertas barreras estructurales y funcionales como son: la falta de objetivos terapéuticos operativos, la descoordinación con y entre recursos socio-sanitarios, la limitada comunicación interprofesional entre los dispositivos de salud general y las unidades de conductas adictivas, las carencias de formación del personal sanitario y el insuficiente uso de instrumentos para la valoración de los resultados de los programas (Rodriguez Reinado, Millan Carrasco, Venegas Sanchez y March Cerda, 2010). 


\section{Conclusiones}

Actualmente, la metadona es el fármaco de primera línea indicado con evidencias nivel A y con grado de recomendación nivel I indicado para el tratamiento de la adicción a opiáceos más empleado en todo el mundo, además es uno de los que mayor evidencia empírica ha obtenido a largo plazo en términos de eficacia y efectividad. Entre las fortalezas de los programas de mantenimiento con metadona se han atribuido la reducción del consumo de opiáceos, menor tasa de actividad criminal, mejora de la comorbilidad psiquiátrica y física, y una mayor tasa de retención en los programas, sin embargo, como debilidades se han establecido la sedación, la disminución del funcionamiento cognoscitivo y los riesgos cardiovasculares como consecuencia de la alteración del ritmo cardíaco. Estos factores influyen indirectamente en una disminución de la carga pública de esta enfermedad crónica, tanto a nivel de utilización de recursos socio-sanitarios como en costes globales.

Desafortunadamente no existen muchas evidencias del tamaño del efecto que puedan determinar la psicoterapia grupal o individual en la evolución a largo plazo de estos pacientes. Por otra parte, tampoco existe suficiente nivel de evidencia del impacto evolutivo de la comorbilidad física o psiquiátrica en las evaluaciones a largo plazo. Con este nivel de evidencias y las limitaciones de evaluación mencionadas, se sigue considerando crucial que las pautas terapéuticas psicosociales y farmacológicas estén personalizadas (estado de salud, tolerabilidad y deseos coparticipados del paciente).

\section{Conflicto de intereses}

Los autores declaran que no existe ningún conflicto de interés.

\section{Referencias}

Amato, L., Davoli, M., Perucci, C. A., Ferri, M., Faggiano, F. y Mattick, R. $P$. (2005). An overview of systematic reviews of the effectiveness of opiate maintenance therapies: available evidence to inform clinical practice and research. Journal Substance Abuse Treatment, 28, 321-329.

Amato, L., Minozzi, S., Davoli, M., y Vecchi, S. (2011). Psychosocial combined with agonist maintenance treatments versus agonist maintenance treatments alone for treatment of opioid dependence. Cochrane Database Systematic Review, 10, CD004147.

Anchersen, K., Clausen, T., Gossop, M., Hansteen, V., y Waal, H. (2009). Prevalence and clinical relevance of corrected OT interval prolongation during methadone and buprenorphine treatment: a mortality assessment study. Addiction, 104, 993-999.
Arango, C. (2012). Evolución de la investigación psiquiátrica en España. Algo más que brotes verdes. Revista de Psiquiatría y Salud Mental, 6, 210-212.

Arroyo, A., Marrón, T., Coronas, D., Leal, M. J., Solé, C., y Laliaga, A. (2000). Programa de mantenimiento con metadona (PMM) en prisión: Cambios sociales y sanitarios. Adicciones, 12, 187-194.

Bao, Y. P., Du, C., Lu, H. Y., Lian, Z., Qiu, Y., Mu, Y., et al. (2012). The investigation of HIV and HCV infection and risk factors among opiate drug users in Beijing, China. American Journal of Drug and Alcohol Abuse, 38, 140-145.

Bobes-Bascaran, M. T., Bascaran, M. T., Saiz, P. A., Garcia-Portilla, M. P., y Bobes, J. (2012). Long-term outcomes of pharmacological treatments of opioid dependence: Does methadone still lead the pack? British Journal of Clinical Pharmacology, 74 (in press)

Casares-Lopez, M. J., Diaz-Mesa, E. M., Garcia-Portilla, M. P., Saiz, P. A., Bobes-Bascaran, M. T., Fonseca-Pedrero, E., et al. (2011). Sixth version of the Addiction Severity Index: Assessing sensitivity to therapeutic change and retention predictors. International Journal of Clinical and Health Psychology, 11, 495-508.

Coviello, D. M., Zanis, D. A., Wesnoski, S. A., Lynch, K. G., y Drapkin, M. (2011). Characteristics and 9-month outcomes of discharged methadone maintenance clients. Journal Substance Abuse Treatment, 40, 165-174.

Diaz-Mesa, E. M., Garcia-Portilla, M. P., Saiz, P. A., Bobes-Bascaran, M. T., Casares, M. J., Fonseca, E., et al. (2010). Psychometric performance of the $6^{\text {th }}$ version of the Addiction Severity Index in Spanish (ASI-6). Psicothema, 22, 513-519.

Dolan, K. A., Shearer, J., White, B., Zhou, J., Kaldor, J., y Wodak, A. D. (2005). Four-year follow-up of imprisoned male heroin users and methadone treatment: mortality, re-incarceration and hepatitis $\mathrm{C}$ infection. Addiction, 100, 820-828.

Fernandez Miranda, J. J. (2005). Efectividad de los programas de mantenimiento con metadona. Adicciones, 17, 167-180.

Grella, C. E. y Lovinger, K. (2012). Gender differences in physical and mental health outcomes among an aging cohort of individuals with a history of heroin dependence. Addictive Behaviors, 37, 306-312.

Iraurgi Castillo, I. (2007). Comparación del instrumento de salud SF-12 frente al SF-36 en pacientes con mantenimiento con metadona. Adicciones, 19, 56-67.

Jimenez-Trevino, L., Saiz, P. A., Garcia-Portilla, M. P., Diaz-Mesa, E. M., Sanchez-Lasheras, F., Buron, P., et al. (2011). A 25-year follow-up of patients admitted to methadone treatment for the first time: mortality and gender differences. Addictive Behavior, 36, 11841190.

Kleber, H. D. (2008). Methadone maintenance 4 decades later: thousands of lives saved but still controversial. JAMA, 300, 23032305.

Lingford-Hughes, A. R., Welch, S., Peters, L. y Nutt, D. J. (2012). Evidence-based guidelines for the pharmacological management of substance abuse, harmful use, addiction and comorbidity: recommendations from BAP. Journal of Psychopharmacology (en prensa). 
Maremmani, I. y Gerra, G. (2010). Buprenorphine-based regimens and methadone for the medical management of opioid dependence: selecting the appropriate drug for treatment. American Journal on Addictions, 19, 557-568.

Martinez Delgado, M., Fernández-Repeto Guilloto, M. y Ramírez López, J. (2005). Tratamientos farmacológicos del abuso y dependencia de opiáceos. In C. p. I. I. y. B. Social. (Ed.), Guía de práctica clínica basada en la evidencia para el manejo de la adicción a opiáceos. Sevilla: Junta de Andalucía.

Mateu, G., Astals, M. y Torrens, M. (2005). Comorbilidad psiquiátrica y trastorno por dependencia de opiáceos: del diagnóstico al tratamiento. Adicciones, 17, 111-121.

National Institute for Health and Clinical Excellence. Methadone and buprenorphine for the management of opioid dependence. (2007). NICE, Technology appraisal guidance 114.

Nosyk, B., Marsh, D. C., Sun, H., Schechter, M. T. y Anis, A. H. (2010). Trends in methadone maintenance treatment participation, retention, and compliance to dosing guidelines in British Columbia, Canada: 1996-2006. Journal of Substance Abuse Treatment 39, 22-31.

Nosyk, B., Sun, H., Evans, E., Marsh, D. C., Anglin, M. D., Hser, Y. I., et al. (2012). Defining dosing pattern characteristics of successful tapers following methadone maintenance treatment: Results from a population-based retrospective cohort study. Addiction.

Oliver, P., Keen, J., Rowse, G., Ewins, E., Griffiths, L., y Mathers, N. (2010). The effect of time spent in treatment and dropout status on rates of convictions, cautions and imprisonment over 5 years in a primary care-led methadone maintenance service. Addiction, 105, 732-739.

Pani, P. P., Maremmani, I., Pacini, M., Lamanna, F., Maremmani, A. G. y Dell'osso, L. (2011). Effect of psychiatric severity on the outcome of methadone maintenance treatment. European Addiction Research 17, 80-89.

Randall, D., Degenhardt, L., Vajdic, C. M., Burns, L., Hall, W. D., Law, M., et al. (2011). Increasing cancer mortality among opioiddependent persons in Australia: a new public health challenge for a disadvantaged population. Australian and New Zealand Journal of Public Health, 35, 220-225.

Reed, G. M., y Ayuso-Mateos, J. L. (2011). Hacia una clasificación Internacional de los Trastornos Mentales de la OMS de mayor utilidad clínica. Revista de Psiquiatría y Salud Mental, 4, 113-116.

Rodriguez Reinado, C., Millan Carrasco, A., Venegas Sanchez, J., March Cerda, J. C. (2010). [Opportunities for the improvement of the methadone service in primary health care, from the professionals' point of view]. Adicciones, 22, 207-215.

Rosca, P., Haklai, Z., Goldberger, N., Zohar, P., Margolis, A., Ponizovsky, A. M. (2012). Mortality and causes of death among users of methadone maintenance treatment in Israel, 1999-2008. Drug and Alcohol Dependence (en prensa).

Schafer, I., Eiroa-Orosa, F. J., Verthein, U., Dilg, C., Haasen, C. y Reimer, J. (2010). Effects of psychiatric comorbidity on treatment outcome in patients undergoing diamorphine or methadone maintenance treatment. Psychopathology, 43, 88-95.
Soyka, M., Kranzler, H. R., van den Brink, W., Krystal, J., Moller, H. J. y Kasper, S. (2011). The World Federation of Societies of Biological Psychiatry (WFSBP) guidelines for the biological treatment of substance use and related disorders. Part 2: Opioid dependence. World Journal of Biological Psychiatry, 12, 160-187.

Torrens Melich, M. y Fonseca Casals, F. (2009). La eficacia de los tratamientos sustitutivos con opioides. Revisión de la evidencia científica. In Tratamiento de mantenimiento con metadona: Manual de práctica clínica. Barcelona: Subdirección General de Drogodependencias. Generalitat de Catalunya. Departamento de Salud.

Trujols, J., Garijo, I., Sinol, N., Del Pozo, J., Portella, M. J. y Pérez de Los Cobos, J. (2011). Patient satisfaction with methadone maintenance treatment: The relevance of participation in treatment and social functioning. Drug and Alcohol Dependence, 123, 41-47.

Wang, P. W., Wu, H. C., Yen, C. N., Yeh, Y. C., Chung, K. S., Chang, H. C. y Yen, C. F. (2012). Change in Quality of Life and Its Predictors in Heroin Users Receiving Methadone Maintenance Treatment in Taiwan: An 18-Month Follow-Up Study. American Journal of Drug and Alcohol Abuse, 38, 213-219.

Whelan, P. J., y Remski, K. (2012). Buprenorphine vs methadone treatment: A review of evidence in both developed and developing worlds. Journal of Neurosciences in Rural Practice, 3, 45-50.

Zapata, M. A., Torres de, G. Y. y Montoya, L. P. (2012). [Risk of pathological gambling, Associated factors and mental disorders in youth from Medellin - Colombia]. Adicciones, 23, 17-25. 
